\title{
Sleep Duration and Oxidative Stress: The Buffalo Cardio-Metabolic Occupational Police Stress (BCOPS) Study
}

\author{
Luenda E. Charles ${ }^{1} *$, Cecil M. Burchfiel ${ }^{1}$, Desta Fekedulegn ${ }^{1}$, John M. Violanti ${ }^{2}$, Richard W. \\ Browne $^{2}$, Erin McCanlies ${ }^{1}$ and Michael E. Andrew
}

\begin{abstract}
${ }^{I}$ Biostatistics and Epidemiology Branch, Health Effects Laboratory Division, National Institute for Occupational Safety and Health, Centers for Disease Control and Prevention, Morgantown, WV; ${ }^{2}$ School of Public Health and Health Professions, Department of Social and Preventive Medicine, State University of New York at Buffalo, USA
\end{abstract}

\begin{abstract}
Our objective was to investigate the cross-sectional association between sleep duration and biomarkers of oxidative stress (glutathione (GSH), glutathione peroxidase (GSH-Px), vitamin C, thiobarbituric acid reactive substances (TBARS), and trolox equivalent antioxidant capacity (TEAC)) among police officers. Standardized techniques were used to analyze biomarkers in fasting blood specimens. Mean levels of biomarkers were compared across levels of sleep duration (0-4.9, 5.0-6.9, $\geq 7.0$ hours) using ANOVA. Officers' (women=44; men=69) mean age was 39.6 years. Positive trends were observed across sleep categories with mean levels of GSH and vitamin C only among women ( $\mathrm{p}$ trend=0.156 and 0.022 respectively), with attenuation after risk-factor adjustment. Positive trends were observed for vitamin $\mathrm{C}$ among older officers ( $p$ trend $=0.018$ ) but not younger. No associations were observed between sleep duration and biomarkers among men. Longer sleep duration is associated with higher levels of vitamin C among policewomen and older officers. Additional studies are warranted.
\end{abstract}

Key Words: Sleep, glutathione peroxidase, vitamin C, oxidative stress, thiobarbituric acid reaction substances, trolox equivalent antioxidant capacity.

\section{INTRODUCTION}

The United States is a sleep-deprived nation [1]. Adequate sleep is necessary for survival and good health. Sleep deprivation is associated with adverse effects on the immune, cardiovascular, and endocrine systems [2-5], and with impaired moral judgments and decision making [6,7]. Sleep deprivation (in animal experiments) and obstructive sleep apnea syndrome (in human populations) are also associated with increased oxidative stress [8-11]. In a laboratory experiment, rats that were deprived of sleep for 5 or 10 days were observed to have decreased glutathione levels (i.e., increased oxidative stress) which returned to normal within 48 hours of recovery sleep [12].

Oxidative stress is defined as an imbalance between the production and removal of reactive oxygen/nitrogen species and is associated with several adverse outcomes such as cancers, immunodeficiency diseases, inflammation, neurological diseases, and cardiovascular diseases [13-15]. Environmental factors such as cigarette smoke can increase oxidative stress [16]. The plasma concentration of thiobarbituric acid reaction substances (TBARS) has been widely used to measure oxidative stress levels $[17,18]$. Antioxidants such as glutathione (GSH) and vitamin $\mathrm{C}$ remove free radicals and decrease oxidative stress levels, thereby protecting tissues $[13,15,19]$. Glutathione peroxidase (GSH-Px) is an antioxidant enzyme and counteracts oxidative stress by reducing hydrogen peroxide and alkyl hydroperoxides $[13,20]$. Trolox equivalent antioxidant capacity (TEAC) is a biomarker of

*Address correspondence to this author at the National Institute for Occupational Safety and Health, HELD/BEB, MailStop L-4050, 1095 Willowdale Rd., Morgantown, WV 26505-2888, USA; Tel: (304) 285-5922; Fax:(304) 285-6112; E-mail: 1charles@cdc.gov antioxidant capacity in peripheral blood [21]. Reduced antioxidant capacity is an index of excessive oxidative stress.

Police officers are likely to be sleep-deprived due to exposure to multiple occupational stressors including shift work and long work hours [22,23]. Therefore, if short sleep duration is associated with increased oxidative stress which increases the risk of certain diseases, sleep deprivation may increase the risk of chronic diseases among police officers. The objective of this investigation was to examine the association of sleep duration with levels of five biomarkers of oxidative stress (GSH, GSH-Px, vitamin C, TBARS, TEAC) and a summary variable of oxidative stress status among this occupational group.

\section{MATERIALS AND METHODOLOGY}

In this cross-sectional study, a random sample of 115 police officers was selected in 1999 from all officers in the Buffalo, New York, police department using a computergenerated random sample table. All of these officers agreed to participate in the study. Women were over-sampled. Data were collected at the Center for Preventive Medicine, School of Public Health and Health Professions, State University of New York at Buffalo [23]. Informed consent was obtained from all participants. This study was approved by the State University of New York at Buffalo Internal Review Board and the National Institute for Occupational Safety and Health Human Subjects Review Board. After excluding officers with missing data on the main variables, the final sample size was 113 officers, 44 women and 69 men.

\section{Assessment of Sleep Duration}

Officers were asked how many hours, on average, they slept each 24-hour period during the previous five weekdays 
(i.e., Sunday through Thursday) and during the previous weekend (i.e., Friday and Saturday nights). The hours of sleep reported for the two periods were averaged to give the total hours of sleep per 24-hour period during the previous seven days.

\section{Assessment of Biomarkers of Oxidative Stress}

Analyses of GSH, GSH-Px, vitamin C, TBARS, and TEAC were performed by staff (R.W.B.) at the State University of New York at Buffalo on blood collected from participants who had fasted for 12 hours.

Glutathione. Erythrocyte GSH was measured in EDTA whole blood using the method by Browne and Armstrong (1998) [24], after extraction and stabilization in metaphosphoric acid within 1 hour of phlebotomy. Clear supernatant was decanted and frozen at $-76^{\circ} \mathrm{C}$. Results are expressed as milligrams of GSH per deciliter of packed red blood cells by applying the original whole blood hematocrit.

Glutathione peroxidase. GSH-Px was measured in heparinized plasma by the enzyme kinetic assay on the Cobas Mira automated chemistry analyzer using glutathione reductase and NADPH [25].

Vitamin C. Sample pretreatment was carried out within 1 hour of phlebotomy. Samples for analysis were stabilized by adding $0.5 \mathrm{~mL}$ heparin plasma to $2.0 \mathrm{ml} 6 \%$ MPA and centrifuging at 3,000 $\mathrm{X} \mathrm{g}$ for 10 minutes. Clear supernatant was decanted and frozen at $-76^{\circ} \mathrm{C}$. Measurements of vitamin $\mathrm{C}$ was performed on the heparinized plasma and was determined by the 2,4-dinitrophenyl hydrazine method [26].

TBARS. TBARS were measured in EDTA plasma by a modification of the method of Armstrong and Browne (1994) [27] and expressed in $\mathrm{nmol} / \mathrm{ml}$ malondialdehyde (MDA) equivalents.

$T E A C$. EDTA plasma was used to measure TEAC by the method of Miller et al. (1993) [21] and is expressed as a percent inhibition of the radical generating reaction relative to the vitamin $\mathrm{E}$ analogue trolox.

\section{Assessment of Covariates}

Police officers completed questionnaires (self- and interviewer-administered) that elicited information on demographic characteristics, lifestyle behaviors, and medical history. Body mass index (BMI) was calculated as weight in kilograms divided by height in meters squared. Officers were asked how often they consumed alcoholic beverages: 12-oz. can or bottle of beer, 1 medium glass of wine, and 1 shot of liquor. The total number of drinks per month (of each type) was summed and then divided by 4 to give the approximate total number of drinks consumed per week. Participants reported their smoking status as current, former, or never. The Center for Epidemiologic Studies Depression (CES-D) Scale was used to measure depressive symptoms because of its validity and high reliability [28]. This 20 -item scale was designed to measure depressive symptoms in the general population and is commonly used to assess the relationship between depression and other variables.

Police officers reported the duration (hours per week, hours per weekend) and intensity (moderate, hard, very hard) of three types of physical activity (occupational, household, and sports) that they engaged in during the previous seven days. A total physical activity score was then computed by summing the intensities of the three types (i.e., occupational, household, and sports) of physical activity performed during the weekday and the weekend. Intensity score was computed as the product of number of hours and intensity of physical activity, where moderate, hard, and very hard levels were assigned intensities of 1,2, and 3, respectively. An objective measure of shift-work was developed from the daily payroll records of officers for the 1994-1000 time period. Officers were classified into one of three shifts based on the highest percentage of hours worked on each of these shifts using the following start times: day shift, start time between 0400 and 1100 hours; afternoon shift, start time between 1200 and 1900 hours; and midnight shift, start time between 2000 and 0300 hours.

\section{Statistical Methods}

Characteristics of participants were described using univariate statistical methods. Pearson's correlation coefficients were used to assess the bivariate relationships between the antioxidants and oxidative stress markers, sleep duration, and demographic, anthropometric, and lifestyle variables. TBARS had a slight non-normal distribution and so, was log-transformed. Analysis of the original and logtransformed TBARS produced similar results therefore we presented the results using the original form of TBARS. Analysis of variance and covariance (ANOVA and ANCOVA) were used to determine the unadjusted and riskfactor adjusted associations between sleep duration and the biomarkers of oxidative stress. In addition, a summary measure of oxidative stress [29] referred to as oxidative stress score (OSS) was created using the following variables: blood levels of GSH, GSH-Px, Vitamin C, TBARS and TEAC; smoking status; and use of at least one type of antiinflammatory medications (e.g., ibuprofen), nutritional supplements (e.g., selenium, fish oil), and herbal products (e.g., black cohosh, milk thistle). Each variable was dichotomized using the respective median as the cut point. The two categories were then coded 0 or 1 where 1 represents the group with higher oxidative stress. For example, participants with GSH, GSH-Px, vitamin C, and TEAC values below the median were coded 1 for each marker; for TBARS, values above the median were coded 1 . Persons who reported that they had used (in the previous 30 days) at least one antiinflammatory medication, vitamins or supplements, or herbal products were given a code of 0 for each of these variables. For smoking, ever smokers (current and former) received a code of 1 . The OSS was obtained by summing the dummy codings for the nine variables. Sleep duration was categorized into three groups: $0-4.9,5-6.9$, and $\geq 7$ hours. Trends in unadjusted and risk-factor adjusted mean values of the oxidative stress biomarkers were examined across the three ordinal sleep categories. The multivariate models that assessed association between sleep duration and the biomarkers included these variables as potential confounders: age, BMI, smoking status, alcohol consumption, CES-D scores, and education. The multivariate models of sleep duration and OSS included the same covariates except smoking status as potential confounders. For all associations, effect modification was assessed for smoking status (current and former smokers vs. never smokers), age (26-40 years vs. 41-61 
Table 1. Descriptive Statistics of Demographic and Lifestyle Characteristics, Oxidative Stress Biomarkers and OSS by Gender

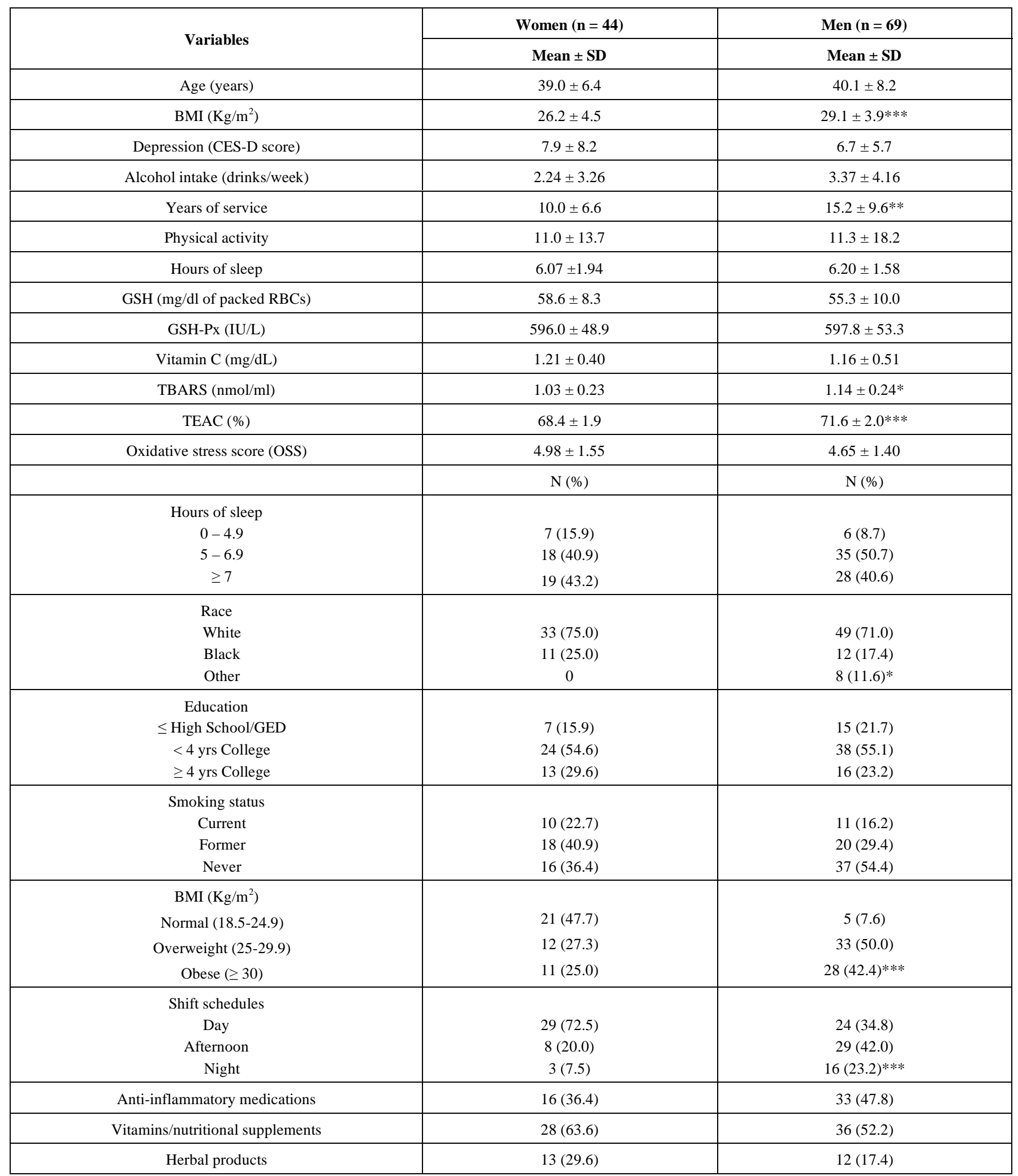

$* \mathrm{p}<0.05 ; * * \mathrm{p}<0.01 ; * * * \mathrm{p}<0.001$.

years), and shift work schedule (day, afternoon, and night shifts). To account for the reduced power, statistical significance for effect modification was set at an alpha level of $<0.200$. Results are presented for all officers and separately for women and men. SAS version 9.1 was used to analyze these data [30]. 


\section{RESULTS}

\section{Descriptive Statistics}

Descriptive statistics of the study participants are provided in Table 1. The officers ranged in age from 26 to 61 years. The mean age of women was 39.0 years $(\mathrm{SD}=6.4)$ and of men, 40.1 years $(\mathrm{SD}=8.2)$. Twenty-nine officers (25.7\%) obtained a college education or higher. Almost $23 \%$ of women were current smokers compared to $16.2 \%$ of men. Both women and men reported approximately 6 hours of sleep per 24-hour period. Compared to women, men had significantly higher mean TBARS levels (1.14 \pm 0.24 vs. $1.03 \pm 0.23 \mathrm{nmol} / \mathrm{ml} ; \mathrm{p}=0.021)$ and significantly higher mean TEAC levels $(71.6 \pm 2.0$ vs. $68.4 \pm 1.9 \%$; p <0.0001) Women had higher mean levels of GSH than men (58.6 \pm 8.3 vs. $55.3 \pm 10.0 \mathrm{mg} / \mathrm{dl})$ but the difference was not statistically significant $(\mathrm{p}=0.073)$.

\section{Correlations Between Main Variables and Covariates}

Bivariate relations between main variables and covariates are shown in Table $\mathbf{2}$. Vitamin C levels were positively correlated with hours of sleep, but the association was significant only among women $(r=0.303 ; \mathrm{p}=0.046)$. Among men, vitamin $\mathrm{C}$ levels were positively and significantly correlated with age $(\mathrm{r}=0.328 ; \mathrm{p}=0.006)$, number of alcoholic drinks per week $(\mathrm{r}=0.271 ; \mathrm{p}=0.024)$, and years of service $(\mathrm{r}=$ $0.386 ; \mathrm{p}=0.001)$. GSH levels were positively correlated with hours of sleep in women $(r=0.271 ; p=0.075)$ GSH and GSH-Px levels were inversely correlated with BMI among women $(\mathrm{r}=-0.370 ; \mathrm{p}=0.014$ and $\mathrm{r}=-0.297, \mathrm{p}=$ 0.050 respectively). Among men, OSS was negatively correlated with alcohol intake $(r=-0.302 ; \mathrm{p}=0.012)$, GSH levels were positively correlated with physical activity $(\mathrm{r}=0.280 ; \mathrm{p}$ $=0.020)$, and sleep duration was inversely correlated with CES-D score $(r=-0.273 ; p=0.029)$.

Correlations between each of the blood antioxidant levels and oxidative stress measures were also examined (data not shown). Among women, TEAC was negatively correlated with GSH $(\mathrm{r}=-0.207$; $\mathrm{p}=0.178)$, GSH-Px $(\mathrm{r}=-0.234 ; \mathrm{p}=$ $0.126)$, and positively correlated with vitamin $C(r=0.226$; $p$ $=0.139$ ). Among men only, a significant positive correlation was observed between TBARS and TEAC: $\mathrm{r}=0.263, \mathrm{p}=$ 0.031 , and a negative correlation was observed between GSH-Px and TBARS: $\mathrm{r}=-0.231, \mathrm{p}=0.056$.

\section{Associations Between Main Variables and Covariates}

Among women, higher educational attainment was positively associated with increasing mean values of vitamin $\mathrm{C}$ ( $\mathrm{p}$ for trend $=0.047$ ) and negatively associated with mean values of TBARS and OSS ( $\mathrm{p}$ for trend $=0.031$ and 0.036 respectively) (Table 3). Among women, never smokers had a significantly higher mean level of vitamin $\mathrm{C}(1.40 \pm 0.30)$ compared to current smokers $(0.88 \pm 0.38), p=0.001$. Also, current smokers had a significantly higher mean OSS $(6.0 \pm$ 1.2) compared to former $(4.6 \pm 1.4)$ or never smokers $(4.4 \pm$

Table 2. Pearson's Correlation Coefficients for Measures of Oxidative Stress Biomarkers, OSS, Hours of Sleep, and Covariates, by Gender

\begin{tabular}{|c|c|c|c|c|c|c|c|}
\hline Variables & GSH & GSH-Px & Vitamin C & TBARS & TEAC & OSS & $\begin{array}{c}\text { Hours of } \\
\text { Sleep }\end{array}$ \\
\hline & \multicolumn{7}{|c|}{ WOMEN (N = 44) } \\
\hline Hours of sleep & 0.271 & 0.139 & $0.303^{*}$ & 0.025 & -0.141 & -0.287 & $\mathrm{Na}$ \\
\hline Age (years) & -0.121 & 0.030 & 0.054 & 0.155 & 0.154 & -0.149 & -0.185 \\
\hline Depression (CES-D score) & -0.070 & -0.180 & 0.020 & -0.124 & -0.068 & -0.036 & -0.168 \\
\hline Alcohol (drinks/wk) & 0.101 & 0.269 & 0.148 & -0.094 & -0.068 & -0.147 & 0.265 \\
\hline Years of service & -0.072 & 0.004 & 0.181 & 0.199 & 0.270 & -0.192 & -0.215 \\
\hline Physical activity & 0.174 & 0.250 & -0.101 & -0.197 & -0.236 & 0.024 & 0.171 \\
\hline Age (years) & -0.094 & -0.106 & $0.328 * *$ & 0.106 & -0.057 & -0.073 & 0.194 \\
\hline BMI $\left(\mathrm{Kg} / \mathrm{m}^{2}\right)$ & 0.030 & $-0.256^{*}$ & -0.101 & 0.019 & 0.126 & 0.041 & -0.091 \\
\hline Depression (CES-D score) & -0.025 & -0.154 & -0.023 & -0.130 & 0.015 & 0.060 & $-0.273^{*}$ \\
\hline Alcohol (drinks/wk) & -0.026 & 0.074 & $0.271^{*}$ & -0.021 & 0.180 & $-0.302 *$ & 0.041 \\
\hline Years of service & -0.048 & -0.085 & $0.386^{* *}$ & 0.089 & -0.007 & -0.162 & 0.171 \\
\hline Physical activity & $0.280^{*}$ & 0.071 & 0.115 & 0.038 & -0.126 & -0.093 & -0.087 \\
\hline
\end{tabular}

$* \mathrm{p}<0.05 ; * * \mathrm{p}<0.01 ; * * * \mathrm{p}<0.001$. 
Table 3. Unadjusted Mean Values of Oxidative Stress Biomarkers and OSS by Levels of Selected Variables, by Gender

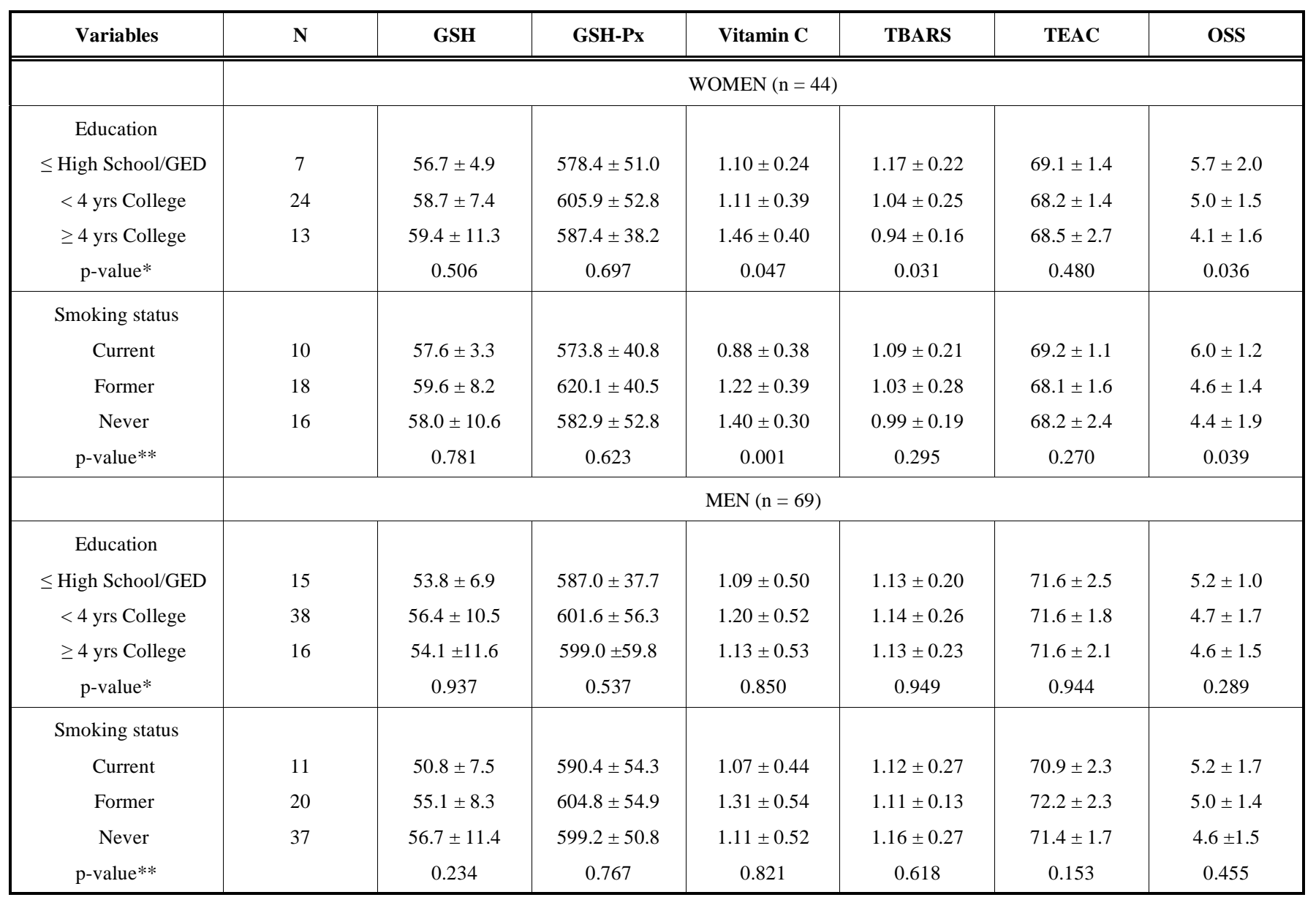

Values are means \pm SDs.; $*$ p-values from polynomial orthogonal contrasts.; $* *$ p-values for differences between the groups.

1.9), $\mathrm{p}=0.039$. Additionally, among women, mean hours of sleep increased with increasing level of educational status ( $p$ for trend $=0.072$ ), and never smokers reported a significantly higher mean sleep duration compared to current smokers $(6.7 \pm 1.3$ vs. $4.6 \pm 2.7 ; p=0.013)$ (data not shown). Among both groups, current and former smokers had significantly higher mean OSS values compared to never smokers $(\mathrm{p}=0.042$ among women; $\mathrm{p}=0.009$ among men).

\section{Associations Between Sleep Duration and Biomarkers}

Multivariate-adjusted mean levels of GSH increased monotonically with increasing hours of sleep among women ( $\mathrm{p}$ for trend $=0.147$ ) $($ Table 4$)$. Unadjusted mean levels of vitamin $\mathrm{C}$ increased with increasing hours of sleep for women $(0.90 \pm 0.49 ; 1.23 \pm 0.37 ; 1.30 \pm 0.36$; $\mathrm{p}$ for trend $=$ 0.022). Adjustment for covariates, especially smoking, attenuated the association between sleep duration and vitamin $\mathrm{C}$ ( $\mathrm{p}$ for trend $=0.214$ ). No associations were found between sleep duration and the other biomarkers. Mean values of OSS decreased with increasing hours of sleep ( $\mathrm{p}$ for trend = 0.090), with attenuation after risk-factor adjustment.

There were no significant associations between sleep duration and the oxidative stress biomarkers or OSS among men (Table 5). However, stepwise positive trends in the mean values of vitamin $\mathrm{C}$ are seen with increasing hours of sleep.
In Table 6, the associations between sleep duration and the biomarkers are presented for all officers. Increasing levels of vitamin $\mathrm{C}$ are observed with increasing hours of sleep and the trend is statistically significant before adjustment for covariates ( $\mathrm{p}$ for trend $=0.015$ ). After adjustment for age, sex, BMI, education, smoking status, alcohol consumption, and CES-D scores, the trend was slightly attenuated and no longer statistically significant ( $\mathrm{p}$ for trend $=0.156$ ). Mean levels of OSS decreased monotonically with increasing hours of sleep but the trends were not statistically significant.

When stratified by smoking status, increasing mean levels of vitamin $\mathrm{C}$ were observed with increasing levels of sleep among both groups, but the trend was only significant among the smokers (data not shown). However, smoking status was not found to be an effect modifier in the association between sleep duration and vitamin $\mathrm{C}$ ( $\mathrm{p}$ for interaction $=0.907)$. Shift work was not found to be an effect modifier in the associations between sleep duration and the biomarkers (data not shown). However, age modified the association between sleep duration and levels of vitamin $\mathrm{C}$ ( $\mathrm{p}$ for interaction $=0.107$ ) (data not shown). Among the younger officers (i.e., 26-40 years), no association was observed between sleep duration and vitamin C. However, among officers who were $\geq 41$ years, there was a significant increase in the mean levels of vitamin $\mathrm{C}$ with increasing hours of sleep: $0.94 \pm$ $0.45,1.21 \pm 0.54$, and $1.53 \pm 0.45(\mathrm{p}$ for trend $=0.018)$. 
Table 4. Unadjusted and Adjusted Mean Values of Oxidative Stress Biomarkers and OSS by Levels of Sleep Duration for Women

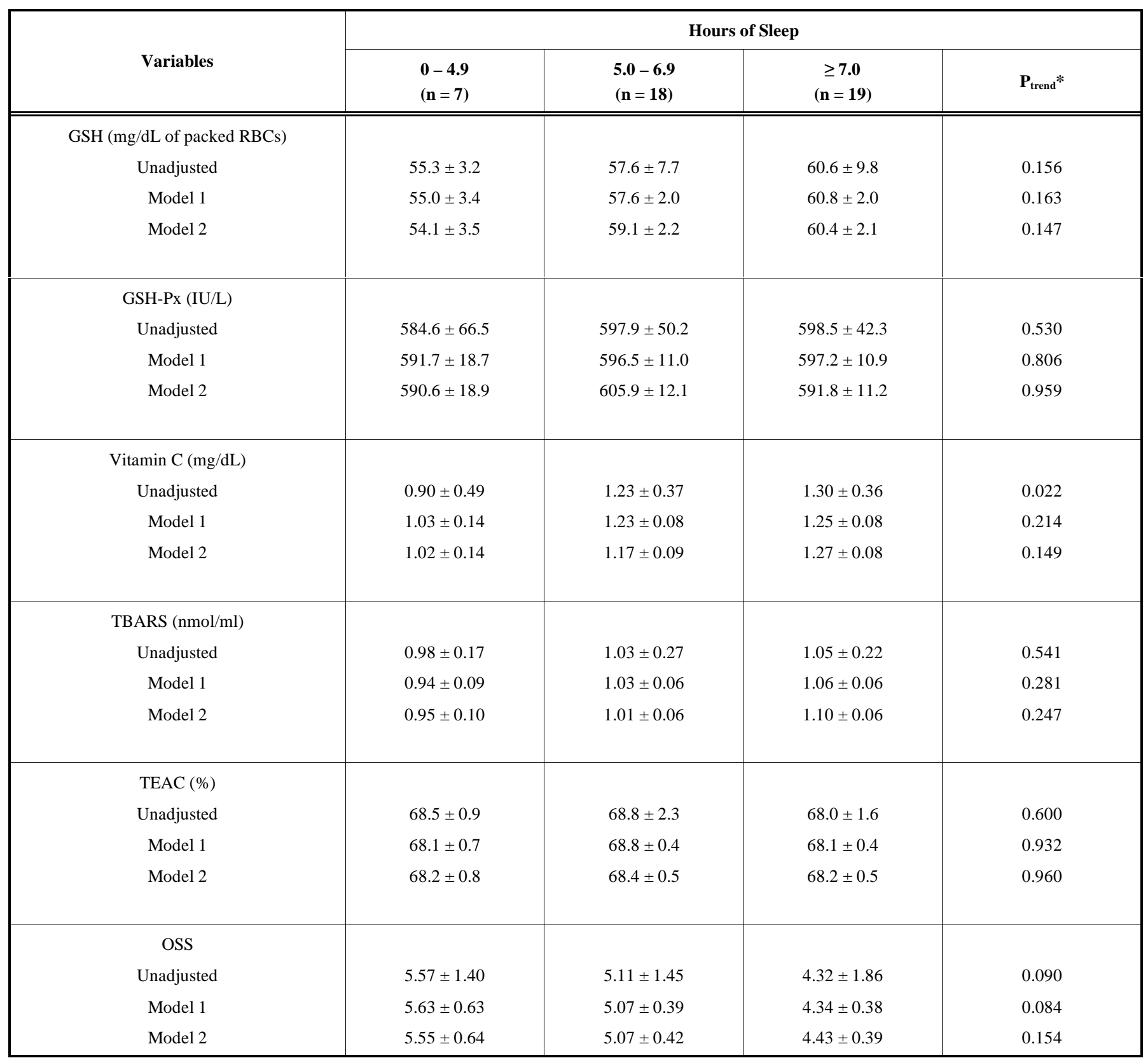

Values are means \pm SD for unadjusted models and means \pm SE for adjusted models.; *p-values from polynomial orthogonal contrasts; For all models except OSS:- Model 1: Adjusted for smoking status; Model 2: Adjusted for age, BMI, education, smoking status, CES-D score, and alcohol consumption.; For OSS:- Model 1: Adjusted for BMI; Model 2: Adjusted for age, BMI, education, CES-D score, and alcohol consumption.

\section{DISCUSSION}

Several epidemiologic studies have investigated the association between sleep disorders such as obstructive sleep apnea syndrome and antioxidant status and have reported lower total antioxidant status in patients compared to controls [8,31]. To our knowledge, our study is the first epidemiologic study to investigate the association between sleep duration and oxidative stress. Results from our study indicated that mean blood/plasma levels of the antioxidants GSH and vitamin $\mathrm{C}$ were positively associated with duration of sleep among women. Mean levels of OSS were inversely associated with sleep duration among women. After adjustment for several potential confounders, these associations were attenuated but the stepwise trends remained. We did not find any associations for sleep duration with GSH-Px, TBARS, or TEAC among women. Among men, there was a positive stepwise trend between sleep duration and vitamin C which is note-worthy even in the absence of a significant $\mathrm{p}$ value. When women and men were combined, age was an effect modifier in the association between sleep duration and vitamin $\mathrm{C}$, with older officers showing a significant positive association. It is possible that sleep is more important for maintenance of antioxidant status as a person gets older.

We had expected to find an inverse association between sleep duration and blood levels of TBARS. Chronic sleep deprivation constitutes a form of allostatic load and, in ani- 
Table 5. Unadjusted and Adjusted Mean Values of Oxidative Stress Biomarkers and OSS by Levels of Sleep Duration for Men

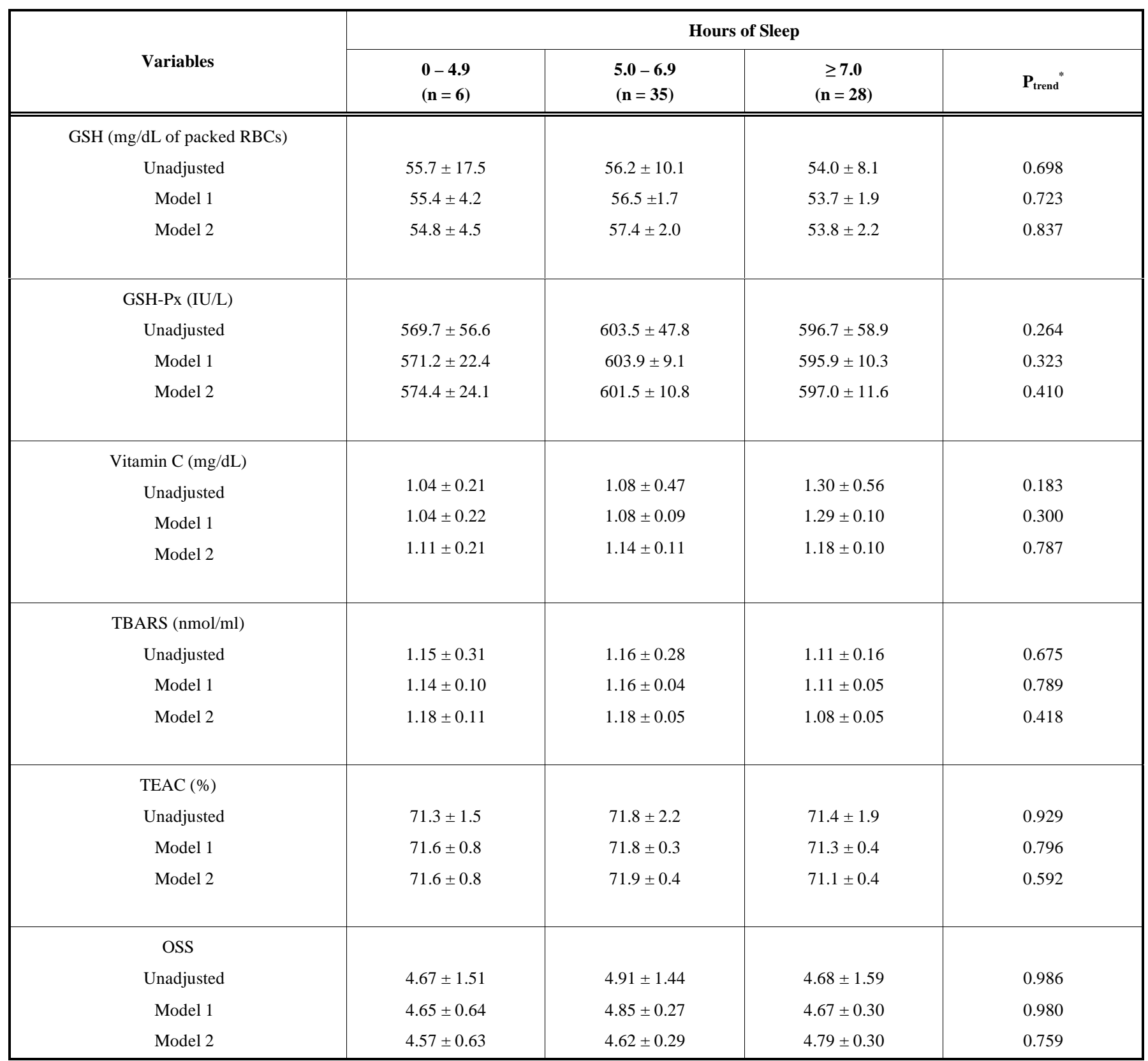

Values are means \pm SD for unadjusted models and means \pm SE for adjusted models; *p-values from polynomial orthogonal contrasts; For all models except OSS:- Model 1: Adjusted for smoking status; Model 2: Adjusted for age, BMI, education, smoking status, CES-D score, and alcohol consumption.; For OSS:- Model 1: Adjusted for BMI; Model 2: Adjusted for age, BMI, education, CES-D score, and alcohol consumption.

mal studies, has been shown to cause several adverse health outcomes including increased oxidative stress [10]. Short sleep duration negatively influences the oxidant-antioxidant system via its effect on metabolic and endocrine function [32-34]. Furthermore, sleep deprivation is associated with elevations of plasma cortisol in the evening [35], a phenomenon which is associated with aging [36] and, by inference, increased oxidative stress.

Our analyses also showed that, among women, higher levels of educational attainment was positively associated with vitamin $\mathrm{C}$ and negatively associated with TBARS and OSS, and mean hours of sleep increased with increasing level of educational status. Not surprisingly, it appears that officers who were more educated engaged in lifestyle behaviors that were conducive to better health with consequential lower oxidative stress. Also among women, never smokers had a higher mean level of vitamin $\mathrm{C}$ compared to current smokers, and reported a higher mean number of hours of sleep and a lower mean OSS compared to current smokers. The lack of association between sleep duration and TBARS is unexpected. However, according to Halliwell and Whiteman (2004), use of TBARS as a biomarker of lipid peroxidation is strongly discouraged [37]. They recommended use of malondialdehye (MDA) as an improvement to TBARS and, even though they are not ideal, the isoprostanes as the best 
Table 6. Unadjusted and Adjusted Mean Values of Oxidative Stress Biomarkers and OSS by Levels of Sleep Duration for all Officers

\begin{tabular}{|c|c|c|c|c|}
\hline Variables & \multicolumn{4}{|c|}{ Hours of Sleep } \\
\hline \multicolumn{5}{|l|}{$\mathrm{GSH}$ (mg/dL of packed RBCs) } \\
\hline Unadjusted & $55.5 \pm 11.5$ & $56.7 \pm 9.3$ & $56.7 \pm 9.3$ & 0.708 \\
\hline Model 1 & $54.8 \pm 2.6$ & $56.8 \pm 1.3$ & $56.7 \pm 1.4$ & 0.518 \\
\hline \multicolumn{5}{|l|}{ GSH-Px (IU/L) } \\
\hline Unadjusted & $577.7 \pm 60.0$ & $601.6 \pm 48.2$ & $597.4 \pm 52.3$ & 0.223 \\
\hline Model 1 & $576.9 \pm 14.5$ & $601.4 \pm 7.1$ & $597.9 \pm 7.6$ & 0.201 \\
\hline Model 2 & $584.8 \pm 14.7$ & $603.9 \pm 7.8$ & $593.5 \pm 7.9$ & 0.612 \\
\hline \multicolumn{5}{|l|}{ Vitamin $\mathrm{C}(\mathrm{mg} / \mathrm{dL})$} \\
\hline Unadjusted & $0.94 \pm 0.45$ & $1.13 \pm 0.44$ & $1.30 \pm 0.47$ & 0.015 \\
\hline Model 1 & $0.95 \pm 0.13$ & $1.14 \pm 0.06$ & $1.28 \pm 0.07$ & 0.023 \\
\hline Model 2 & $1.01 \pm 0.13$ & $1.16 \pm 0.07$ & $1.22 \pm 0.07$ & 0.156 \\
\hline \multicolumn{5}{|l|}{ TBARS (nmol/ml) } \\
\hline Unadjusted & $1.06 \pm 0.25$ & $1.12 \pm 0.28$ & $1.08 \pm 0.19$ & 0.773 \\
\hline Model 1 & $1.08 \pm 0.07$ & $1.11 \pm 0.03$ & $1.08 \pm 0.03$ & 0.976 \\
\hline Model 2 & $1.10 \pm 0.07$ & $1.12 \pm 0.04$ & $1.08 \pm 0.04$ & 0.795 \\
\hline \multicolumn{5}{|l|}{$\operatorname{TEAC}(\%)$} \\
\hline Unadjusted & $69.8 \pm 1.9$ & $70.7 \pm 2.6$ & $70.0 \pm 2.4$ & 0.740 \\
\hline Model 1 & $70.3 \pm 0.5$ & $70.6 \pm 0.3$ & $70.1 \pm 0.3$ & 0.774 \\
\hline Model 2 & $70.3 \pm 0.5$ & $70.5 \pm 0.3$ & $69.9 \pm 0.3$ & 0.521 \\
\hline \multicolumn{5}{|l|}{ OSS } \\
\hline Unadjusted & $5.08 \pm 1.71$ & $4.94 \pm 1.41$ & $4.51 \pm 1.44$ & 0.218 \\
\hline Model 1 & $5.02 \pm 0.41$ & $4.96 \pm 0.20$ & $4.51 \pm 0.21$ & 0.274 \\
\hline Model 2 & $5.02 \pm 0.40$ & $4.77 \pm 0.22$ & $4.63 \pm 0.22$ & 0.402 \\
\hline
\end{tabular}

Values are means \pm SD for unadjusted models and means \pm SE for adjusted models; * ${ }^{*}$-values from polynomial orthogonal contrasts; For all models except OSS:- Model 1: Adjusted for smoking status; Model 2: Adjusted for age, BMI, education, smoking status, CES-D score, and alcohol consumption; For OSS:- Model 1: Adjusted for BMI; Model 2: Adjusted for age, BMI, education, CES-D score, and alcohol consumption

available biomarkers of lipid peroxidation. In our study, neither MDA nor the isoprostanes were measured.

In our study, BMI was inversely related to GSH and GSH-Px, whereas levels of TBARS were not associated with smoking status or alcohol consumption among women. Similar results were reported in the population-based study by Trevisan and colleagues (2001) [18]. The results of our study also showed significantly higher levels of TBARS and TEAC in men compared to women. Some studies investigating the association between oxidative stress and diseases reported similar gender differences in these markers [18,38]. Ochs-Balcom et al. (2005) [38] reported that men had higher
TBARS levels than did women, indicating increased oxidative stress or decreased antioxidant capacity. However, results from the Epidémiologie du Vieillissement Artériel study conducted in France showed that women had higher levels of TBARS compared to men [39]. The gender disparity in these results may be partially due to a hormonal effect. Estrogens act as antioxidants [40], although in some situations they may act as prooxidants [41]. The results of a Polish study showed that the administration of natural estrogens to postmenopausal women diminished oxidative stress and increased antioxidative cell potency [42]. According to Vina et al. (2006) [40], estrogens do not act as chemical antioxi- 
dants in vivo but apply their antioxidant effect by upregulating the expression of antioxidant genes and enzymes.

Studies have shown associations between individual biomarkers of oxidative stress and many health conditions. In one study, among men, lung function was inversely associated with TBARS and glutathione, and positively associated with glutathione peroxidase [38]. In another study, TBARS levels were significantly higher in those with prevalent CVD compared to those without a CVD diagnosis, after adjusting for CVD risk factors [17]. Low levels of vitamin C have been found in cancer patients $[43,44]$ and persons with dementia [45]. In another population-based study, oxidative status and antioxidant potentials were significantly associated with a number of major cardiovascular disease risk factors [18].

Even though ingestion of single doses of antioxidants have not been shown to be effective against DNA damage or oxidative stress in some randomized clinical trials [46], other studies have shown that administration of antioxidants can reverse some of the damage caused by oxidative stress. Grebe et al. (2006) [47] found that vitamin C was found to improve flow-mediated dilation (FMD) of the brachial artery among untreated patients with obstructive sleep apnea. Parenteral administration of high doses of vitamin $C$ has been shown to reduce the toxic side-effects of chemotherapy [48] and may restore vascular function in critically ill patients [19]. In a clinical study, improvements in patients with Parkinson's disease were observed following administration of reduced GSH [49].

Limitations in this study include the sample size and the cross-sectional design that precludes causal inferences. Our use of TBARS instead of MDA or the isoprostanes is also a limitation. It has been reported that oxidative stress and certain biomarkers vary with time of day [37] and we did not take into account the time of day of blood collection. Another limitation is that although we adjusted for several confounders, there may have been additional potential confounders that we failed to adjust for because they were not available. The oxidative stress score variable that we created is imperfect since we did not have information on specific times used nor on the quantity of supplements, herbs, and anti-inflammatory medications taken by the participants. We may have included persons with very different consumption patterns in the same group. In addition, information on sleep was obtained only for the prior week and, for some officers, may not have been representative of their regular sleeping habits. The effects of any non-differential biases due to an imperfect OSS variable or to misclassification of sleep duration would result in dilution of the respective associations. Although it may appear as a limitation, we do not believe that the subjective reporting of sleep duration resulted in bias because studies comparing actigraphic monitoring with selfreported data have shown good congruency between the two methods [50,51].

Imaki and colleagues (2002) [52] found that Japanese factory workers who failed to get sufficient sleep were more likely to engage in poor dietary habits such as irregularity in eating and consumption of insufficient quantities of fruits and vegetables that may increase oxidative stress. Nutrient intake is associated with socio-economic status [53] and investigators in France recommended that socio-economic factors and lifestyle habits be taken into consideration when investigating studies of antioxidant status [39]. Therefore, we controlled for educational attainment.

Strengths of the study include precise measurements of the oxidative stress biomarkers and $100 \%$ participation of the randomly selected officers. Although studies have investigated the association between obstructive sleep apnea and oxidative stress, to our knowledge, this is the first study to investigate the association of sleep duration with oxidative stress. A unique strength of our study was the incorporation of a summary variable of oxidative stress status [29], which included the antioxidants, TBARS, and use of supplements, herbs, and anti-inflammatory medications which would influence the antioxidant/oxidant system. We were able to adjust for several factors which may be potential surrogates for the officers' dietary habits, such as education, smoking status, and BMI. It is possible that adjustment for these variables may yield even more accurately estimated antioxidant/oxidant status than adjustment for dietary intake for which time of collection is imprecise. Finally, even though this study was conducted among police officers, these results may be generalizable to persons in different yet related occupations such as other first responders and health care providers.

\section{CONCLUSION}

Our results show that sleep duration was positively associated with levels of vitamin $\mathrm{C}$, and after adjustment for other covariates, particularly smoking, this association was attenuated. Stratification by age also revealed a significant association between sleep duration and vitamin C levels only among the older officers. Sleep duration was inversely associated with OSS among women only, although this trend was not statistically significant. Further studies investigating the association between sleep duration and oxidative stress are recommended and may be enhanced by use of a prospective study design, larger sample sizes, and additional biomarkers of oxidative stress such as the isoprostanes.

\section{ACKNOWLEDGEMENTS}

This work was supported by the National Institute for Occupational Safety and Health (NIOSH), contract no. HELD01B0088. The findings and conclusions in this article are those of the authors and do not necessarily represent the views of the National Institute for Occupational Safety and Health.

\section{REFERENCES}

[1] National Sleep Foundation 2005 sleep in America poll. Washington DC: National Sleep Foundation.

[2] Carskadon MA. Sleep deprivation: health consequences and societal impact. Med Clin N Am 2004; 88: 767-776.

[3] Copinschi G. Metabolic and endocrine effects of sleep deprivation. Essent Psychopharmacol 2005; 6: 341-347.

[4] Everson CA. Functional consequences of sustained sleep deprivation in the rat. Behav Brain Res 1995; 69: 43-54.

[5] Gangwisch JE, Heymsfield SB, Boden-Albala B, et al. Short sleep duration as a risk factor for hypertension: analyses of the first $\mathrm{Na}$ tional Health and Nutrition Examination Survey. Hypertension 2006; 47: 833-839.

[6] Killgore WDS, Killgore DB, Day LM, Li C, Kamimori GH, Balkin TJ. The effects of 53 hours of sleep deprivation on moral judgment. Sleep 2007; 30: 345-352. 
[7] Killgore WDS, Balkin TJ, Wesenten NJ. Impaired decision making following 49 hours of sleep derpivation. J Sleep Res 2005; 14: 1-7.

[8] Barcelo A, Barbe F, de la Pena M, et al. Antioxidant status in patients with sleep apnoea and impact of continuous positive airway pressure treatment. Eur Respir J 2006; 27: 756-760.

[9] Lavie L. Obstructive sleep apnoea syndrome - an oxidative stress disorder. Sleep Med Rev 2003; 7: 35-51.

[10] McEwen BS. Sleep deprivation as a neurobiologic and physiologic stressor: allostasis and allostatic load. Metab Clin Exp 2006; 55: S20-S23.

[11] Schulz R, Mahmoudi S, Hattar K, et al. Enhanced release of superoxide from polymorphonuclear neutrophils in obstructive sleep apnea. Impact of continous positive airway pressure therapy. Am J Respir Crit Care Med 2000; 162: 566-570.

[12] Everson CA, Laatsch CD, Hogg N. Antioxidant defense responses to sleep loss and sleep recovery. Am J Physiol Regul Integr Comp Physiol 2005; 288: R374-R383.

[13] Townsend DM, Tew KD, Tapiero H. The importance of glutathione in human disease. Biomed Pharmacother 2003; 57: 145155.

[14] Turrens JF. Mitochondrial formation of reactive oxygen species. J Physiol 2003; 552: 335-344.

[15] Wu G, Fang YZ, Yang S, Lupton JR, Turner ND. Glutathione metabolism and its implication for health. J Nutr 2004; 34: 489492.

[16] Isik B, Ceylan A, Isik R. Oxidative stress in smokers and nonsmokers. Inhal Toxicol 2007; 19: 767-769.

[17] Schisterman EF, Faraggi D, Browne R, et al. TBARS and cardiovascular disease in a population-based sample. J Cardiovasc Risk 2001; 8: 219-225.

[18] Trevisan M, Browne R, Ram M, et al. Correlates of markers of oxidative status in the general population. Am J Epidemiol 2001; 154: 348-356.

[19] McGregor GP, Biesalski HK. Rationale and impact of vitamin C in clinical nutrition [micronutrients]. Curr Opin Clin Nutr Metab Care 2006; 9: 697-703.

[20] Brigelius-Flohe R. Tissue-specific functions of individual glutathione peroxidases. Free Radic Biol Med 1999; 27: 951-965.

[21] Miller NJ, Rice-Evans C, Davies MJ, Gopinathan V, Milner A. A novel method for measuring antioxidant capacity and its application to monitoring the antioxidant status in premature neonates. Clin Sci 1993; 84: 407-412.

[22] Vila B. Impact of long work hours on police officers and the communities they serve. Am J Ind Med 2006; 49: 972-980.

[23] Violanti JM, Burchfiel CM, Miller DB, et al. The Buffalo CardioMetabolic Occupational Police Stress (BCOPS) Pilot Study: Methods and Participant Characteristics. Ann Epidemiol 2006; 16: 148156.

[24] Browne R, Armstrong D. Fluorometric determination of glutathione and glutathione disulfide. In: Armstrong D, ed. Methods in Molecular Biology, Free Radicals and Antioxidant Protocols. Totowa, New Jersey: The Humana Press, Inc., 1998; 347-52.

[25] Pippenger CE, Browne R, Armstrong D. Regulatory antioxidant enzymes. In: Armstrong D, ed. Methods in Molecular Biology, Free Radicals and Antioxidant Protocols. Totowa, New Jersey: The Humana Press, Inc., 1998; 299-313.

[26] Burtis C, Ashwood E. Dinitrophenyl hydrazine Method. In: Burtis C, Ashwood E, eds. Tietz Textbook of Clinical Chemistry 2nd Edition; W.B. Saunders Co. 1994; 1313-314.

[27] Armstrong D, Browne R. The analysis of free radicals, lipid peroxides, antioxidant enzymes and compounds related to oxidative stress as applied to the clinical chemistry laboratory. In: Armstrong D, ed. Free Radicals in Diagnostic Medicine. Adv. Exp. Med. Biol. Vol. 366. New York: Plenum Press Corp. 1994; 43-58.

[28] Radloff LS. The CES-D Scale: A self-report depression scale for research in the general population. Appl Psychol Meas 1977; 1: $385-401$.

[29] Goodman M, Bostick RM, Dash C, Flanders WD, Mandel JS. Hypothesis: Oxidative stress score as a combined measure of pro- oxidant and antioxidant exposures. Ann Epidemiol 2007; 17: 394399.

[30] SAS Institute, Inc. SAS user's guide, version 9.1. Cary NC: SAS Institute, Inc, 2001-2004.

[31] Christou K, Moulas AN, Pastaka C, Gourgoulianis KI. Antioxidant capacity in obstructive sleep apnea patients. Sleep Med 2003; 4: 225-228.

[32] Irwin M, Thompson J, Miller C, Gillin C, Ziegler M. Effects of sleep and sleep deprivation on catecholamine and interleukin-2 levels in humans: Clinical implications. J Clin Endocrinol Metab 1999; 84: 1979-1985.

[33] Redwine L, Hauger RL, Gillin C, Irwin M. Effects of sleep and sleep deprivation on interleukin-6, growth hormone, cortisol, and melatonin levels in humans. J Clin Endocrinol Metab 2000; 85: 3597-3603.

[34] Spiegel K, Leproult R, Van Cauter E. Impact of sleep debt on metabolic and endocrine function. Lancet 1999; 354: 1435-1439.

[35] Leproult R, Copinschi G, Buxton O, Van Cauter E. Sleep loss results in an elevation of cortisol levels the next evening. Sleep 1997; 20: 865-870.

[36] Van Cauter E, Leproult R, Kupfer DJ. Effects of gender and age on the levels and circadian rhythmicity of plasma cortisol. J Clin Endocrinol Metab 1996; 81: 2468-2473.

[37] Halliwell B, Whiteman M. Measuring reactive species and oxidative damage in vivo and in cell culture: how should you do it and what do the results mean? Br J Pharmacol 2004; 142: 231-255.

[38] Ochs-Balcom HM, Grant BJ, Muti P, et al. Oxidative stress and pulmonary function in the general population. Am J Epidemiol 2005; 162: 1137-1145.

[39] Berr C, Coudray C, Bonithon-Kopp C, Roussel A, Mainard F, Alperovitch A, and the EVA study Group. Demographic and cardiovascular risk factors in relation to antioxidant status: the EVA study. Int J Vit Nutr Res 1998; 68: 26-35.

[40] Vina J, Sastre J, Pallardo FV, Gambini J, Borras C. Role of mitochondrial oxidative stress to explain the different longevity between genders. Protective effect of estrogens. Free Radic Res 2006; 40: 1359-1365.

[41] Nathan L, Chaudhuri G. Antioxidant and prooxidant actions of estrogens: potential physiological and clinical implications. Semin Reprod Endocrinol 1998; 16: 309-314.

[42] Bednarek-Tupikowska G, Tworowska U, Jedrychowska I, et al. Effects of oestradiol and oestroprogestin on erythrocyte antioxidative enzyme system activity in postmenopausal women. Clin Endocrinol 2006; 64: 463-468.

[43] Mayland CR, Bennett MI, Allan K. Vitamin C deficiency in cancer patients. Palliat Med 2005; 19: 17-20.

[44] Saygili EI, Konukoglu D, Papila C, Akcay T. Levels of plasma vitamin $\mathrm{E}$, vitamin $\mathrm{C}$, TBARS, and cholesterol in male patients with colorectal tumors. Biochemistry 2004; 68: 325-328.

[45] Charlton KE, Rabinowitz TL, Geffen LN, Dhansay MA. Lowered plasma vitamin $\mathrm{C}$, but not vitamin $\mathrm{E}$, concentration in dementia patients. J Nutr Health Aging 2004; 8: 99-107.

[46] Choi SW, Benzie IFF, Collins AR, Hannigan BM, Strain JJ. Vitamins $\mathrm{C}$ and $\mathrm{E}$ : acute interactive effects on biomarkers of antioxidant defence and oxidative stress. Mut Res 2004; 551: 109-117.

[47] Grebe M, Eisele HJ, Weissmann N, et al. Antioxidant vitamin C improves endothelial function in obstructive sleep apnea. Am J Respir Crit Care Med 2006; 173: 897-901.

[48] Shimpo K, Nagatsu T, Yamada K, et al. Ascorbic acid and adriamycin toxicity. Am J Clin Nutr 1991; 54: 1298S-13101S.

[49] Sechi G, Deledda MG, Bua G, et al. Reduced intravenous glutathione in the treatment of early Parkinson's disease. Prog Neuropsychopharmacol Biol Psych 1996; 20: 1159-1170.

[50] Kushida CA, Chang A, Gadkary C, Guilleminault C, Carrillo O, Dement WC. Comparison of actigraphic, polysomnographic, and subjective assessment of sleep parameters in sleep-disordered patients. Sleep Med 2001; 2: 389-396. 
[51] Lockley SW, Skene DJ, Arendt J. Comparison between subjective and actigraphic measurement of sleep and sleep rhythms. J Sleep Res 1999; 8: 175-183.

[52] Imaki M, Hatanaka Y, Ogawa Y, Yoshida Y, Tanada S. An epidemiological study on relationship between the hours of sleep and life style factors in Japanese factory workers. J Physiol Anthropol Appl Human Sci 2002; 21: 115-120.

[53] Bolton-Smith C, Smith WCS, Woodward M, Tunstall-Pedoe H.

Nutrient intakes of different social-class groups: results from the Scottish Heart Health Study (SHHS). Br J Nutr 1991; 65: 321-335. 